\title{
O processo de trabalho na Estratégia Saúde da Família voltado às pessoas com sobrepeso e obesidade em São Paulo
}

\author{
Work process in the Family Health Strategy oriented to people with \\ overweight and obesity in São Paulo
}

Juliana Giaj Levra de Jesus'1, Célia Maria Sivalli Campos', Fernanda Baeza Scagliusi', Luciene Burlandy², Cláudia Maria Bógus ${ }^{1}$

DOI: $10.1590 / 0103-1104202213212$

RESUMO O objetivo do artigo é analisar as características dos processos de trabalho na Estratégia Saúde da Família direcionados às pessoas com sobrepeso e obesidade no município de São Paulo. Ancorado no referencial teórico da saúde coletiva sobre processo de trabalho, foi desenvolvido um estudo exploratório com abordagem qualitativa. Foram realizadas onze entrevistas com profissionais de uma unidade básica de saúde, na zona leste da cidade de São Paulo, entre os meses de julho e agosto de 2019. A partir da análise temática, o artigo apresenta os resultados e a discussão em três categorias analíticas: objeto de trabalho, instrumentos de trabalho e organização do trabalho. O cuidado às pessoas com sobrepeso e obesidade é mediado pelo baixo investimento em qualificação dos profissionais de saúde sobre o tema, pela falta de materiais técnicos de suporte ao trabalho e pelo modelo de gestão pautado pela produtividade e cobrança de resultados quantitativos. Conclui-se que os profissionais de saúde apresentam pouco domínio sobre seu processo de trabalho, cuja finalidade acaba sendo a mudança de comportamento e a perda de peso corporal, em vez de considerar as necessidades de saúde dos usuários e profissionais, produzindo, por vezes, insatisfação e desgaste com o trabalho.

PALAVRAS-CHAVE Manejo da obesidade. Atenção Primária à Saúde. Qualidade da assistência à saúde. Condições de trabalho. Sistema Único de Saúde.

ABSTRACT The goal of the article is to analyze the characteristics of the work processes in the Family Health Strategy directed to people with overweight and obesity in the city of São Paulo. Based on the theoretical framework of collective health about work processes, an exploratory study with a qualitative approach was developed. Eleven interviews were conducted with professionals from a basic health unit, in the east of the city of São Paulo, between July and August 2019. Starting with the thematic analysis, the article presents the results and discussion in three analytical categories: work object, work instruments and work organization. The care for people with overweight and obesity is mediated by the low investment in training health professionals on the subject, the lack of technical materials to support the work and the management model based on productivity and demand for quantitative results. It is concluded that health professionals have little control over their work process, whose purpose ends up being the change of behavior and the loss of

1 Universidade de São Paulo (USP) - São Paulo (SP), Brasil.

jujulevra@gmail.com

2 Universidade Federal Fluminense (UFF) - Niterói (RJ), Brasil. body weight, instead of considering the health needs of users and professionals, sometimes producing dissatisfaction and weariness with work.

KEYWORDS Obesity management. Primary Health Care. Quality of health care. Working conditions. Unified Health System. 


\section{Introdução}

Como espaço de primeiro contato dos usuários com o Sistema Único de Saúde (SUS) no País, a organização da Atenção Primária à Saúde (APS) tem como modelo prioritário de assistência a Estratégia Saúde da Família (ESF)' $\mathbf{1}$. Diversos autores ressaltam que a expansão da ESF nos últimos 30 anos representa o crescimento dos cuidados primários da população, o aumento na oferta de serviços em regiões periféricas e rurais e a redução das internações por causas sensíveis à $\mathrm{APS}^{1-3}$. Também destacam que o modelo de assistência da ESF busca contrapor-se ao modelo biomédico e promover um trabalho multidisciplinar baseado em vínculo, acolhimento, orientação comunitária e princípios do SUS1,3-5.

Apesar disso, são diversos os desafios para a efetivação da ESF no SUS com a flexibilização da priorização política para fortalecimento desse modelo de assistência, sobrecarga dos profissionais de saúde, falta de formação para o trabalho, financiamento insuficiente e cortes de recursos destinados ao SUS,4,6-11. Somam-se a isso o envelhecimento populacional e a piora das condições de trabalho, com aumento das taxas de desemprego e de empregos informais ${ }^{12}$, que impactam os parâmetros de saúde e alimentação da população.

A perda da qualidade do que se consome, com o aumento da ingestão de alimentos ultraprocessados (com alto valor calórico e baixo valor nutricional), em detrimento dos alimentos in natura ou de preparações caseiras, tem se relacionado com o aumento de diabetes, hipertensão arterial sistêmica e insegurança alimentar e nutricional, tanto no perfil de carências nutricionais como de sobrepeso e obesidade na população ${ }^{\mathbf{1 3}, 14}$. A Organização Mundial da Saúde (OMS) considera a obesidade uma epidemia e um problema de saúde pública mundial, por isso ela tem sido prioridade das políticas públicas nas últimas décadas, principalmente devido à sua relação com o desenvolvimento de outras doenças crônicas, como as cardiovasculares. No Brasil, com relação ao sobrepeso e à obesidade (Índice de
Massa Corporal - IMC maior ou igual a 25kg/ $\mathrm{m}^{2}$ ), destaca-se que, em 2019, a prevalência de pessoas adultas com sobrepeso e obesidade chegou a $61,7 \%$ e $26,8 \%$, respectivamente ${ }^{15}$.

Apesar de existir um consenso na literatura sobre atrelar a crescente prevalência de sobrepeso e obesidade a diversas dimensões (política, econômica, social, cultural), ainda é complexa a compreensão sobre a relação entre esses múltiplos fatores e a elaboração de propostas de intervenção $0^{16}$. Nacionalmente, o Ministério da Saúde tem elaborado documentos orientadores de práticas de cuidado, como o 'Caderno de Atenção Básica nº 38 Obesidade'17; e o Protocolo Clínico e Diretrizes Terapêuticas (PCDT) para Sobrepeso e Obesidade em Adultos ${ }^{18}$. Já no município de São Paulo, o Protocolo de Atendimento da Linha de Cuidado de Sobrepeso e Obesidade busca subsidiar os profissionais de saúde e qualificar a atenção nutricional na Rede de Atenção à Saúde (RAS) ${ }^{19}$.

Diante dos desafios apresentados, este artigo busca evidenciar as condições de trabalho oferecidas aos profissionais de saúde na ESF para o cuidado das pessoas com sobrepeso e obesidade em uma grande cidade como São Paulo, a fim de fornecer subsídios para a qualificação dos processos de trabalho no SUS em realidades semelhantes. Assim, o objetivo do artigo é analisar as características dos processos de trabalho na ESF direcionados às pessoas com sobrepeso e obesidade no município de São Paulo.

\section{Metodologia}

Foi realizado um estudo de caso de abordagem qualitativa em uma Unidade Básica de Saúde (UBS) localizada na zona leste do município de São Paulo. Essa UBS foi selecionada pois tinha a ESF como forma de organização do processo de trabalho, contava com uma equipe do Núcleo Ampliado de Saúde da Família (Nasf) e teve sua participação no estudo aprovada pela Secretaria Municipal de Saúde. 
A UBS estudada contava com cinco equipes de Saúde da Família (eSF), sendo que, durante o período da pesquisa, duas equipes estavam incompletas com ausência do profissional de medicina. Além disso, a UBS contava com três equipes de saúde bucal, um Agente de Promoção Ambiental (APA) e uma equipe do Nasf, composto por um nutricionista, um psicólogo, um assistente social, um fonoaudiólogo, um terapeuta ocupacional, um fisioterapeuta e um ginecologista. Desde 2016, a gestão da UBS e a contratação dos profissionais é indireta, ou seja, a partir da parceria público-privada, com a contratualização do município com uma Organização Social da Saúde (OSS).

Entre julho e agosto de 2019, foi realizada a coleta de dados, inicialmente, com um período de aproximação da pesquisadora ao campo, por meio de observação participante e, posteriormente, com a realização das entrevistas semiestruturadas com os profissionais. Foram selecionados profissionais com formação em nível superior (médicos e enfermeiras das eSF) e profissionais que coordenavam grupos educativos com usuários voltados para questões relacionadas com alimentação e perda de peso na unidade (profissionais do Nasf e do APA). O roteiro de entrevistas foi estruturado em cinco dimensões, sendo elas: perfil dos entrevistados, rotina de trabalho, concepção sobre sobrepeso e obesidade, formação profissional e processo de trabalho destinado a pessoas com sobrepeso e obesidade.

As entrevistas e a observação da rotina de trabalho na UBS pretenderam compreender mais sobre como está organizado o processo de trabalho das eSF, a partir da identificação das atividades desenvolvidas para o cuidado das pessoas com sobrepeso e obesidade, os instrumentos utilizados nesse cuidado, a percepção dos profissionais de saúde sobre a rotina de trabalho e o conhecimento desses profissionais sobre o excesso de peso e a obesidade. Os relatos obtidos nas entrevistas foram submetidos à técnica de análise temática ${ }^{20}$.

A partir do conceito de processo de trabalho em saúde 21, foram definidas previamente categorias analíticas para guiarem a análise dos dados. As categorias representam os componentes do processo de trabalho e serão apresentadas em: (1) Objeto do trabalho: objeto ou sujeito que recebe a intervenção a partir das práticas de cuidado ofertadas; (2) Instrumentos de trabalho: inclui as práticas de cuidado realizadas no serviço de saúde, o conhecimento dos profissionais de saúde sobre sobrepeso e obesidade e os materiais técnicos de suporte utilizados; e (3) Organização do trabalho: modelo de gestão, modelo assistencial na ESF e a finalidade do trabalho realizado.

A fim de preservar a identidade dos entrevistados, as extrações das entrevistas foram codificadas em E (1 a 5) para as enfermeiras, M (1 a 3) para os médicos, $\mathrm{N}$ (1 a 3) para profissionais do Nasf e do APA. O estudo foi aprovado pelo Comitê de Ética em Pesquisa da Faculdade de Saúde Pública da Universidade de São Paulo e pelo Comitê de Ética em Pesquisa da Secretaria Municipal de Saúde de São Paulo. Todos os participantes assinaram o termo de consentimento livre e esclarecido.

\section{Resultados}

Foram realizadas onze entrevistas com profissionais da UBS, sendo a maioria mulheres (81,8\%), enfermeiras $(45,4 \%)$, com contratação no regime de Consolidação das Leis do Trabalho (CLT) (90,9\%) e com tempo na UBS variando entre 3 meses e 15 anos. O perfil dos participantes está detalhado no quadro 1 . 
Quadro 1. Perfil dos profissionais entrevistados segundo código, sexo, idade, tempo de formação, especialização, tempo na UBS, trajetória prévia e tipo de vinculação. São Paulo, 2019

\begin{tabular}{|c|c|c|c|c|c|c|c|}
\hline Cód. & Sexo & Idade & $\begin{array}{l}\text { Tempo de } \\
\text { formação* }\end{array}$ & $\begin{array}{l}\text { Especialização/pós saúde pública ou } \\
\text { saúde coletiva ou saúde da família }\end{array}$ & Tempo na UBS* & $\begin{array}{l}\text { Trajetória prévia na } \\
\text { saúde pública? }\end{array}$ & Tipo de vinculação \\
\hline E1 & $\mathrm{F}$ & 47 anos & 16 anos & Sim & 15 anos & Sim & CLT \\
\hline E2 & $\mathrm{F}$ & 38 anos & 8 anos & Sim & 7 meses & Sim & CLT \\
\hline E3 & $\mathrm{F}$ & 50 anos & 18 anos & $\operatorname{Sim}$ & 8 anos & Sim & CLT \\
\hline E4 & $\mathrm{F}$ & 43 anos & 16 anos & Sim & 11 meses & Sim & CLT \\
\hline E5 & $\mathrm{F}$ & 49 anos & 16 anos & Sim & 15 anos & Sim & CLT \\
\hline M1 & $\mathrm{F}$ & 40 anos & 9 anos & Sim & 3 meses & Sim & $\begin{array}{l}\text { Programa Mais } \\
\text { Médicos }\end{array}$ \\
\hline M2 & M & 28 anos & 1,5 anos & Não & 1 ano e 2 meses & Não & CLT \\
\hline M3 & M & 29 anos & 1 ano & Não & 6 meses & Não & CLT \\
\hline N1 & $\mathrm{F}$ & 31 anos & 10 anos & Não & 4 anos & Não & CLT \\
\hline N2 & $\mathrm{F}$ & 32 anos & 8 anos & Sim & 3 anos & Não & CLT \\
\hline N3 & $\mathrm{F}$ & 28 anos & 7 anos & Não & 4 anos & Sim & CLT \\
\hline
\end{tabular}

Fonte: elaboração própria.

*Cálculo até agosto de 2019.

\section{Objeto e instrumentos de trabalho}

Durante dois meses acompanhando a rotina de trabalho na UBS, notou-se que essa não se diferenciava muito da rotina preconizada para as eSF em âmbito nacional. A rotina dos profissionais, organizada por uma agenda feita pela gestão local, preservava a lógica de organização voltada ao atendimento das linhas de cuidado, especialmente com a produção de consultas e grupos. Apesar de a organização da ESF ser baseada no atendimento às linhas de cuidado, os profissionais relataram desconhecer a linha de cuidado de sobrepeso e obesidade do município.

As principais atividades desenvolvidas eram consultas individuais, reuniões de equipe, visitas domiciliares e grupos. As consultas com médico ou enfermeiro, assim como com os profissionais do Nasf, foram consideradas pelos entrevistados um espaço de cuidado às pessoas com sobrepeso e obesidade. Além disso, os grupos foram apontados como uma via importante para o cuidado dos usuários com sobrepeso e obesidade, especialmente o grupo intitulado 'Na medida', organizado pela nutricionista.

Na UBS estudada, ainda que o cuidado compartilhado entre eSF e Nasf fosse feito majoritariamente de forma fragmentada - casos de dor para o fisioterapeuta, de sobrepeso e obesidade para nutricionista, de sofrimento psíquico para o psicólogo -, a equipe Nasf foi reconhecida pelos profissionais da eSF como um suporte. Os entrevistados consideravam que os profissionais Nasf, com saberes específicos, apoiavam o cuidado dos casos em que os profissionais da eSF apresentavam mais dificuldade de trabalhar, ampliando, assim, a capacidade de cuidado e qualificando a atenção à saúde prestada. O fluxo de atendimento a pessoas com sobrepeso e obesidade que chegavam ao serviço, especialmente nos casos de IMC mais alto e com comorbidade, passava, na sua maioria, pelos cuidados de algum profissional da equipe Nasf, demonstrando a importância dessa equipe no cuidado das pessoas com sobrepeso e obesidade.

Apesar de a semana típica de trabalho ser composta por diversas atividades que incluíam 
os usuários com sobrepeso e obesidade, isso não necessariamente estava atrelado à qualidade do que é ofertado. De maneira geral, os profissionais reconheceram a falta de material informativo fornecido pela própria UBS e que muitas vezes utilizavam recursos próprios para a elaboração de materiais. "Folder aqui não tem, mas eu sei que tem um dos cadernos que tem a questão da obesidade e tem a nutricionista que a gente sempre pode reportar" (E1).

Apenas a nutricionista do Nasf apontou que utilizava o 'Guia Alimentar para a População Brasileira' como material de apoio.

Uma médica apresentou outro ponto como fragilidade para o cuidado das pessoas com sobrepeso e obesidade: a ausência de protocolo. Em seu relato, apontou que isso desestimula a priorização do cuidado, como se apenas os casos que tivessem protocolos estabelecidos fossem os casos que deveriam ser acompanhados:

Eu não tenho um tempo nem um ambiente onde eu possa colocar os obesos, ainda não foi estabelecido um protocolo. Como não tem um protocolo que te respalde, você não vai cumprir tanto aquilo. (M1).

Apesar dos limites ligados ao uso descontextualizado dos protocolos, a presença desse instrumento na APS apareceu como um fator indutor de conduta e subsídio para a tomada de decisão dos profissionais em meio a um cenário de intensificação do trabalho e falta de formação.

Na UBS estudada, o tempo destinado à educação em serviço ocupava um espaço reduzido na agenda dos profissionais, especialmente dos médicos. Segundo os profissionais entrevistados, os cursos e capacitações que incluíam a temática da obesidade ainda eram poucos e pontuais.

Eu fui em bastante palestra, mas de obesidade em específico não lembro assim de nada. Mas já teve falando de doenças crônicas de uma maneira geral, mas, quando fala muito em doenças crônicas, eles focam em diabetes e pressão alta. (N1).
Ao pedirmos para os profissionais nos falarem sobre algum caso de sobrepeso e obesidade que acompanharam - o primeiro caso que viesse à cabeça deles -, destacaram-se casos complexos e de obesidade grau III que envolviam sofrimento psíquico, casos de obesidade infantil e casos de 'sucesso' em que os usuários atendidos perderam peso. A mensuração de um bom trabalho pareceu ser mais validada pelos próprios profissionais quando culminava com a perda de peso, sendo uma fonte de satisfação profissional e êxito do trabalho realizado.

A fala da enfermeira E1 demonstrou que a satisfação com o trabalho está muito vinculada à mudança de comportamento do usuário:

Eu me sinto chateada por ele não fazer, mas eu não deveria me sentir, porque é uma resposta dele para ele mesmo. Tem pouquíssimos casos que a pessoa não compreende, mas, na grande maioria dos casos, a pessoa sabe que aquilo não tá fazendo bem para ela, mas ela olha para você, nos seus olhos, e fala assim: 'mas nesse momento éo que me deixa feliz'. O profissional consegue chegar atétal lugar, mas, assim, o importante é que você vai também ser assim, que a pessoa entende que aquilo não tá fazendo bem para ela, mas naquele momento ela não tem uma outra coisa que vai fazer tão bem pra ela quanto comer, quanto não sair de casa, e a gente vai tentando fazer com que ela faça diferente. (E1).

Ao serem questionados sobre vias para melhoria do cuidado das pessoas com sobrepeso e obesidade na APS, os profissionais entrevistados fizeram algumas sugestões. Entre as sugestões relacionadas ao processo de trabalho, destacaram-se: a necessidade de mais espaços de capacitação dos profissionais de saúde sobre sobrepeso e obesidade; contratação de mais profissionais de saúde, especialmente profissionais do Nasf, como nutricionista e educador físico; mais investimento em instrumentos para promoção da saúde, como hortas e fortalecimento de uma rede de atenção secundária destinada ao cuidado e ao acompanhamento dos casos de sobrepeso e obesidade. 
Além das sugestões relacionadas ao processo de trabalho na APS, os profissionais apresentaram sugestões que envolviam o setor de educação, emprego e renda e planejamento urbano. As sugestões apresentadas demonstram que os profissionais compreendem o sobrepeso e a obesidade como resultado de múltiplos fatores, e que um cuidado mais integral às pessoas com sobrepeso e obesidade no País precisa passar por melhorias no setor saúde e fora dele. Apesar dessa compreensão, nenhum profissional fez menção à participação social dos usuários e dos próprios profissionais como agentes políticos de transformação.

\section{Organização do trabalho}

Sob o argumento da melhor eficiência, o município de São Paulo definiu o gerencialismo como modelo de gestão e como via para ampliação da APS da cidade em $2001^{22}$. Ao município fica a responsabilidade de regular a execução da política pública de saúde a partir da elaboração dos contratos de gestão. No município de São Paulo, devido à enorme extensão territorial, a parceria público-privada varia conforme divisão administrativa e suas coordenadorias regionais.

O relatório de produção é disponibilizado semestralmente no site da OSS contratada para a gestão da UBS estudada. Na planilha fornecida pela OSS, vê-se que as metas mensais estão direcionadas para as eSF e contam com indicadores de número de consultas de médico ESF, de consultas de enfermagem ESF, de visitas domiciliares dos agentes comunitários de saúde, de consultas individuais, de procedimentos da equipe de saúde bucal e obtidos por cada UBS da rede assistencial.

Apesar de o relatório de produção só trazer esses indicadores, os profissionais também estão submetidos a outras formas de monitoramento do trabalho, como entrega de lista de acompanhamento das linhas de cuidado para a gestão local, metas estabelecidas de vacinação e outros procedimentos; e, para os profissionais do Nasf, meta de grupos e atendimentos por mês.
A cobrança de metas de produção apareceu nas falas dos entrevistados como uma dificuldade encontrada na rotina de trabalho para o cuidado das pessoas com sobrepeso e obesidade, especialmente pelo seu efeito sobre o tempo de atendimento.

Infelizmente, o nosso tempo no SUS, na UBS, é 15 minutos. Não tem tempo de trabalhar isso, o máximo você faz o diagnóstico de obesidade, IMC elevado, mas aí a gente consegue trabalhar em outras áreas, encaminhando para nutrição, psicólogo. (M2).

Além disso, para os profissionais, o trabalho na UBS é dificultado pela alta rotatividade de médicos e pela alta demanda de trabalho.

Quando tem o médico, você consegue dividir. Quando não tem médico, a agenda dobra, enche mais rápido, porque todo mundo quer passar com enfermeiro, só que eu não tenho autonomia para fazer exame, então eu tenho que marcar com outro médico, de outra equipe, assim, acaba demandando um número grande (de atendimentos), porque já estavam faltando médico, e, agora, quase eu não consigo vaga para a minha equipe para poder agendar. (E5).

As metas de produção estabelecidas orientam, em grande medida, as atividades desenvolvidas na rotina de trabalho, uma vez que definem a agenda dos profissionais. O tempo destinado para as consultas é uma consequência do número de consultas esperadas para médicos e enfermeiros, que implica, respectivamente, 15 minutos e 20 minutos para os atendimentos. Ao serem questionados sobre as dificuldades para o acompanhamento de usuários com sobrepeso e obesidade no serviço, os profissionais médicos, especialmente, queixaram-se do tempo destinado às consultas.

Com o tempo destinado às consultas, o processo de trabalho em saúde fica direcionado para a solução das queixas apresentadas pelos usuários atendidos. O médico $\mathrm{M} 3$ apontou que as metas impostas ao seu trabalho alteraram a 
sua prática médica, e foi preciso fazer adaptações e priorização de ações, assim, justificando a lógica da queixa-conduta aplicada nas consultas. O profissional colocou em sua fala a ideia de impossibilidade de mudança no tempo de atendimento, demonstrando sensação de impotência na tomada de decisão. Ao falar sobre as ações desenvolvidas com pessoas com sobrepeso e obesidade, o médico falou sobre as consultas:

$O$ contato que a gente tem é durante a consulta. $E$ durante a consulta, a gente fala assim: 'O senhor tá obeso e tem que fazer isso e isso', mas não tem como a gente elaborar muito sobre isso. De início, quando eu ainda tava desapegado da meta, eu fazia uma orientação um pouquinho melhor: 'Que horas o senhor acorda? Que horas o senhor vai dormir? O que o senhor come de manhã? O que o senhor almoça? Que horas janta? Come alguma coisa no meio da manhã no meio da tarde? O que que é?'. Fazia um cardapiozinho, desenhando um receituário para o paciente poder ver, dividia o prato na metade, dividia a metade da metade [...] mas acaba que na consulta de 15 minutos não dá. Eu via que esses pacientes que atendia no início, muitos deles conseguiram ter resultado de perda de peso, redução de pressão, controle de diabetes, e eles referem que foi muito bom que eles ensinam os outros a fazer do mesmo jeito. (M3).

Segue explicando porque que a prática clínica mudou:

Então, porque nos primeiros meses, eles [gestão] têm uma tolerância porque você tá começando. [...] No primeiro mês, nem agenda eu tinha direito, a agenda era um negócio meio abstrato, então eu tinha mais flexibilidade, e, mesmo assim, era: 'Ah, mas você não bateu a meta né, mas você tá começando, então ok'. Quando acabou a tolerância, aí a coisa ficou mais complicada. Aía gente começa a cortar as coisas. Então, assim, isso era uma coisa que eu fazia com todos os pacientes sobrepeso e obeso, eu fazia essa orientação, cortei. Por exemplo, eu fazia o exame físico mais completo, ausculta cardíaca, avaliava abdômen, apalpava, aí cortei.
Agora, só direciono para queixa, por exemplo, o paciente que não é hipertenso, não é diabético, veio com queixa de dor no ombro, eu só faço exame do ombro. Fica uma coisa bem focada, bem pronto-socorro mesmo. (M3).

A queixa sobre o tempo de consulta foi mais presente entre os médicos do que entre as enfermeiras. Em contrapartida, a rotatividade médica apareceu mais entre as enfermeiras, como uma dificuldade para a organização do processo de trabalho, realização de um cuidado longitudinal e como fator de sobrecarga desses profissionais. Em algumas falas, também foi possível identificar a ideia de vocação, de ajuda e de doação ao trabalho como elementos de satisfação profissional e motivação para o trabalho.

\section{Discussão}

\section{O peso corporal como centro do cuidado e (in)satisfação de trabalhadores com os resultados do trabalho}

A hegemonia da concepção de obesidade como risco à saúde nas políticas que orientam o trabalho no SUS ${ }^{16}$ coloca ao profissional de saúde o papel de normatizar os corpos através de intervenções para prevenção dos riscos à saúde, com enfoque na perda de peso. $\mathrm{O}$ nome dado ao grupo sobre nutrição na UBS estudada ('Na medida') exemplifica o enfoque das práticas de cuidado, colocando o peso corporal como principal objeto de intervenção e critério de acesso. Assim, o processo de trabalho em saúde é orientado pela definição do objeto o peso corporal -, e, muitas, vezes só ganha sentido para os profissionais quando existe uma resolutividade do problema apresentado, ou seja, quando os usuários seguem as recomendações e prescrições feitas pelo profissional ou quando o desfecho do tratamento se aproxima do padrão mais saudável e de menor risco. 
Entretanto, as raízes do desenvolvimento do excesso de peso e da obesidade, determinadas socialmente, não são tomadas como objeto das atividades desenvolvidas, que têm como parte da finalidade a mudança de hábitos de alimentação e atividade física e perda de peso. Tais práticas têm potencial para atender apenas a uma parte das necessidades em saúde dos usuários, fator que pode justificar a dificuldade encontrada pelos profissionais de adesão ao processo terapêutico proposto. A dificuldade de adesão ao tratamento da obesidade pode produzir frustrações nos profissionais de saúde ligadas à sensação de impotência e ao despreparo para lidar com esses casos, e uma culpabilização dos usuários ${ }^{23}$.

Outro aspecto que influencia a satisfação com o trabalho relaciona-se aos padrões de desgaste $^{\mathbf{2 4}}$ dos trabalhadores. Esse padrão está ligado ao tipo de processo de trabalho estabelecido, ao processo de valorização desse trabalho e às cargas de trabalho. As cargas de trabalho, por sua vez, são entendidas como as exigências sobre a organização do trabalho e sobre o trabalhador, tanto com materialidade interna (controle, tensão psíquica etc.) quanto externa (físicas, biológicas etc.). A correlação entres essas cargas gera no corpo do trabalhador uma necessidade de adaptação e, possivelmente, um comprometimento das capacidades biopsíquicas, efetivas ou potenciais para desenvolvimento do trabalho, podendo gerar desgaste e adoecimento ${ }^{24}$.

Logo, elementos vinculados à organização do trabalho - falta de funcionários, excesso de atribuições e carga horária elevada - e à gestão do trabalho - avaliação por produtividade e controle excessivo sobre o trabalho - podem gerar aumento da carga de trabalho dos profissionais da UBS estudada. Biff et al. ${ }^{25}$ identificaram no trabalho de enfermeiras da ESF elementos ligados ao aumento da carga de trabalho, como falta de infraestrutura, déficit de profissionais e excesso de atribuições; e à redução da carga de trabalho, como funcionamento adequado das redes de atenção, a resolutividade nas ações e os instrumentos de trabalho em quantidade e qualidade adequa$\operatorname{dos}^{25}$. Todas essas cargas influenciam a forma como os profissionais trabalham, sua satisfação e, consequentemente, o cuidado ofertado às pessoas com sobrepeso e obesidade.

Com relação à formação dos profissionais de saúde e ao acesso a materiais de apoio ao trabalho, o investimento insuficiente na formação permanente e o distanciamento entre os materiais técnicos que já foram produzidos pela gestão e sua utilização na prática do serviço apontam fragilidades para a produção do cuidado qualificado. Lopes et al. ${ }^{26}$ citam a falta de qualificação profissional sobre obesidade e a ausência de materiais de apoio para a prática dos profissionais de saúde, como barreiras para o cuidado voltado para pessoas com obesidade no SUS.

Nesse sentido, a educação permanente é apontada por autores como uma necessidade da APS brasileira, uma vez que pode possibilitar a reflexão coletiva sobre os processos de trabalho e facilitar a implementação de protocolos específicos de cuidado integral de pessoas com obesidade ${ }^{27}$ e melhorar a qualidade da gestão e da atenção em saúde ${ }^{\mathbf{2 8}}$, através da produção de novos sentidos e significados do cuidado entre os profissionais ${ }^{29}$ e da indução de mudanças das práticas de cuidado ${ }^{30}$.

\section{Modelo de gestão e a organização do cuidado}

Vimos que o modelo da produtividade impregnado na ESF do município influencia o processo de trabalho e, consequentemente, a prática de cuidado dos profissionais. Por mais que não haja a necessidade de abordar a questão alimentar em todas as consultas, a impossibilidade dessa prática, determinada pelo tempo limitado de consulta, mesmo com a necessidade detectada pelo profissional de saúde, induz à secundarização das ações ligadas à promoção da saúde e à alimentação e à nutrição. Nesse sentido, Machado et al.31 apontam que a falta de indução da gestão para 
ações de alimentação e nutrição pelas equipes de saúde pode ser um dos motivos para a baixa realização dessas ações na APS, somando-se a estrutura física inadequada e a falta de educação permanente para incorporação de ferramentas de cuidado.

Nota-se que o modelo assistencial e as atividades ofertadas aos usuários em seu processo de trabalho são influenciados pelo modelo de gestão adotado. Na lógica da produtividade e com o pouco tempo para atendimento, as questões de saúde mais agudas tendem a ser mais olhadas e tratadas, em contraposição às atividades de promoção da saúde e prevenção de doenças. Além disso, nota-se que os profissionais de saúde tendem a associar o cuidado das pessoas com sobrepeso e obesidade como atribuição quase exclusiva do profissional nutricionista ou de educação física, podendo gerar falta de corresponsabilização pelo cuidado dos usuários encaminhados ${ }^{32}$. A busca da produtividade também influencia a forma de interação entre os profissionais, gerando tensões entre uma prática interprofissional mais colaborativa e uma prática mais tradicional, centrada em procedimentos ${ }^{32}$.

No setor saúde, o gerencialismo se expressa na terceirização da execução da política de saúde e gestão do trabalho em saúde para instituições de caráter privado sem fins lucrativos, como Organizações Não Governamentais, Organizações Sociais, Fundações Estatais, entre outros. Ao município fica a responsabilidade de regular a execução da política pública de saúde a partir da contratualização e elaboração dos contratos de gestão. Esta possibilita a flexibilização das relações de trabalho e das formas de contratação, remuneração, qualificação e autonomia dos profissionais 22,33,34.

$\mathrm{O}$ contrato de gestão estabelecido entre a Secretaria Municipal de Saúde de São Paulo e a instituição contratada discorre sobre parâmetros para pagamento e avaliação de desempenho por linha de serviço, indicadores utilizados para a avaliação, metas de produção para eSF e outros acordos e obrigações pactuadas entre contratada e contratante. A partir do mecanismo de gestão de resultado, o repasse financeiro total para a Organização Social de Saúde (OSS) contratada é condicionado ao cumprimento de pelo menos $85 \%$ dos parâmetros estabelecidos no contrato de gestão, e valores inferiores a $85 \%$ sofrem com a perda de orçamento progressivamente.

Destaca-se que a avaliação e o monitoramento do trabalho são fundamentais para a (re)organização do processo de trabalho, como elementos para autorreflexão individual e coletiva, como via para a consolidação dos direitos sociais e participação da sociedade nas decisões públicas. Entretanto, submetidos à lógica da produtividade capitalista e sem a participação dos trabalhadores e usuários na sua elaboração e execução, distanciam-se da sua intencionalidade social e servem como via de controle de gastos e controle sobre os profissionais de saúde ${ }^{33,35}$.

A avaliação por desempenho adotada na saúde é um instrumento de gestão que gera superintensificação do trabalho na busca da 'otimização' dos recursos financeiros e da maior extração da mais valia do setor. Como os custos no setor saúde são majoritariamente ligados à contratação da força de trabalho, especialmente na APS, a contenção dos gastos e a busca de maior eficiência do trabalho recaem sobre os trabalhadores de saúde e seu desempenho. Ou seja, a avaliação do trabalho em saúde, sob o paradigma gerencialista, reduz-se ao controle da produção de atividades quantificáveis, serviços e procedimentos em saúde e responsabilização individual dos profissionais ${ }^{33-35}$.

Souza $^{36}$ contrapõe-se à ideia de que o trabalho em saúde seja um espaço privilegiado para as disputas políticas e mudanças no processo produtivo capitalista, já que os sujeitos envolvidos no ato do cuidado, profissional e usuário, estão submetidos a condições desumanizantes e alienadas em seu processo de trabalho. O trabalho em saúde não segue uma lógica própria, mas reproduz a lógica geral do trabalho, mesmo que o produto desse trabalho seja imaterial, através do cuidado em saúde. Assim, contraditoriamente, quanto maior a 
dedicação dos profissionais de saúde para suprir as necessidades dos usuários, maior a chance de adoecimentos, absenteísmo e desgaste ao tratar com os usuários. Em outras palavras, o cuidado desumanizado é prioritariamente resultado das relações capitalistas de produção, fortemente marcadas pela exploração e alienação do trabalho.

Ainda assim, os profissionais entrevistados carregam fortemente em seu discurso a ideia de trabalho em saúde como um trabalho de ajuda aos outros, de ajuda aos mais 'vulneráveis', demonstrando uma desvinculação entre trabalho em saúde e a categoria trabalho e um distanciamento da noção de acesso aos serviços de saúde como direito da população.

A precariedade das condições de trabalho, sobrecarga de trabalho, número elevado de atendimentos, problemas estruturais, pouca valorização dentro da categoria médica, pouca autonomia para atuação, baixa resolutividade das ações e rede deficitária de suporte nos outros níveis de atenção à saúde são alguns dos fatores ${ }^{37}$ que justificam a rotatividade médica vivenciada na UBS estudada. A mudança constante dos médicos também gera a quebra do trabalho em equipe, sendo necessária uma constante repactuação e reorganização do processo de trabalho ${ }^{37}$.

\section{Considerações finais}

Vimos que os profissionais de saúde têm pouca autonomia e pouco tempo para a definição do seu processo de trabalho, o que acaba acarretando precário domínio do objeto de trabalho e de sua finalidade. Por esse motivo, acabam sendo reprodutores de práticas pré-determinadas e acabam, muitas vezes, frustrados com o trabalho. No caso das atividades voltadas para pessoas com sobrepeso e obesidade, o objeto de trabalho é centralmente o peso corporal, e a finalidade acaba se voltando para perda de peso e mudança do comportamento de risco, mesmo que os profissionais compreendam que a determinação do excesso de peso e da obesidade tem raízes na organização social.

Como instrumentos de trabalho, os profissionais desenvolvem ações de tratamento, promoção e prevenção do sobrepeso e da obesidade, especialmente através das consultas e dos grupos, apesar de demonstrarem insuficiência de formação sobre a temática. Por outro lado, a potência desse trabalho é condicionada à lógica de organização do trabalho na ESF no município, com o modelo gerencialista de gestão orientado pela produtividade e 'otimização' de recursos. Com um histórico de flexibilização da forma de contratação dos profissionais, pautada na busca de melhor utilização dos recursos financeiros, essa organização conduz os trabalhadores de saúde a uma prática de cuidado fragmentada, protocolar e voltada, predominantemente, para o cumprimento das metas de produção estabelecidas.

Nesse cenário, os achados desta pesquisa contribuem para preencher uma lacuna de estudos sobre o processo de trabalho em saúde voltado às pessoas com sobrepeso e obesidade em uma metrópole como São Paulo. Além disso, demonstram que, para a melhoria do cuidado das pessoas com sobrepeso e obesidade no SUS, não basta a capacitação dos profissionais de saúde, sendo fundamental o aprimoramento dos processos de trabalho. São necessárias mudanças estruturais, com mais investimento financeiro para contratação de profissionais, garantia de estrutura física e de materiais de suporte adequados ao trabalho, um modelo de gestão participativo e centrado nas necessidades de saúde da população, com espaços de participação dos trabalhadores nas tomadas de decisão sobre o seu processo de trabalho. Além disso, mais tempo destinado à educação permanente no dia a dia do serviço, de forma a incluir e permitir aprofundamento em temas como promoção da saúde, promoção de alimentação adequada e saudável, aspectos estruturais relacionados com o ambiente alimentar, humanização da 
atenção em saúde, considerando a assistência aos usuários e os processos de trabalho dos profissionais de saúde.

Por fim, por ser um estudo de caso com recorte específico, o artigo apresenta limitações no que diz respeito à universalização dos achados. Assim, destaca-se a necessidade de mais estudos sobre os processos de trabalho na APS e seus determinantes estruturais e acerca dos impactos de tal organização na oferta de um cuidado integral à população.

\section{Referências}

1. Giovanella L, Bousquat A, Schenkman S, et al. Cobertura da Estratégia Saúde da Família no Brasil: o que nos mostram as Pesquisas Nacionais de Saúde 2013 e 2019. Ciênc. Saúde Colet. 2021 [acesso em 2021 dez 21]; 26(supl1):2543-2556. Disponível em: https://doi. org/10.1590/1413-81232021266.1.43952020.

2. Castro MC, Massuda A, Almeida G, et al. Brazil's unified health system: the first 30 years and prospects for the future. Lancet. 2019 [acesso em 2021 dez 21]; 394(10195):345-356. Disponível em: https:// doi.org/10.1016/S0140-6736(19)31243-7.

3. Arantes LJ, Shimizu HE, Merchán-hamann E. Contribuições e desafios da Estratégia Saúde da Família na Atenção Primária à Saúde no Brasil: revisão da literatura. Ciênc. Saúde Colet. 2016 [acesso em 2021 dez 21]; 21(5):1499-1510. Disponível em: https://doi. org/10.1590/1413-81232015215.19602015.

\section{Colaboradores}

Jesus JGL (0000-0003-0166-5568)* e Bógus CM (0000-0003-0925-2741)* contribuíram para elaboração do texto, análise e interpretação dos dados, revisão crítica do conteúdo e aprovação da versão final do manuscrito. Campos CMS (0000-0002-1149-9025)*, Scagliusi FB (0000-0001-7861-8597)* e Burlandy L (0000-0003-0875-6374)* contribuíram para revisão crítica do conteúdo e aprovação da versão final do manuscrito.

4. Fertonani HP, Pires DEP, Biff D, et al. Modelo assistencial em saúde: conceitos e desafios para a atenção básica brasileira. Ciênc. Saúde Colet. 2015 [acesso em 2021 dez 21]; 20(6):1869-1878. Disponível em: https:// doi.org/10.1590/1413-81232015206.13272014.

5. Brasil. Ministério da Saúde. Política Nacional de Atenção Básica. Brasília, DF: Ministério da Saúde; 2017.

6. Reis JB, Harzheim E, Nachif MCA, et al. Criação da Secretaria de Atenção Primária à Saúde e suas implicações para o SUS. Ciênc. Saúde Colet. 2019 [acesso em 2021 dez 21]; 24(9):3457-3462. Disponível em: https://doi.org/10.1590/1413-81232018249.18612019.

7. Mendes A, Carnut L. Novo Modelo de Financiamento para qual Atenção Primária à Saúde? Rev. Doming. 2019 [acesso em 2021 dez 21]; (36):1-11. Disponível em: http://idisa.org.br/domingueira/domingueira-n-36-outubro-2019.
${ }^{*}$ Orcid (Open Researcher and Contributor ID). 
8. Massuda A. Primary health care financing changes in the Brazilian Health System: advance ou setback? Ciênc. Saúde Colet. 2020 [acesso em 2021 dez 21]; 25(4):1181-1188. Disponível em: https://doi. org/10.1590/1413-81232020254.01022020.

9. Peruzzo HE, Bega AG, Lopes APAT, et al. Os desafios de se trabalhar em equipe na estratégia saúde da família. Esc. Anna Nery. 2018 [acesso em $2021 \mathrm{dez}$ 21]; 22(4):e20170372. Disponível em: http://www.revenf.bvs.br/scielo.php?script=sci_arttext\&pid=S1414$-81452018000400205 \& \operatorname{lng}=$ pt.

10. Araújo MBS, Rocha PM. Trabalho em equipe: um desafio para a consolidação da estratégia de saúde da família. Ciênc. Saúde Colet. 2007 [acesso em 2021 dez 21]; 12(2):455-464. Disponível em: https://doi. org/10.1590/S1413-81232007000200022.

11. Scherer MDA, Pires DEP, Jean R. A construção da interdisciplinaridade no trabalho da Equipe de Saúde da Família. Ciênc. Saúde Colet. 2013 [acesso em 2021 dez 21]; 18(11):3203-3212. Disponível em: https://doi. org/10.1590/S1413-81232013001100011.

12. Instituto Brasileiro de Geografia e Estatística. Pesquisa Nacional de Saúde 2019. Rio de Janeiro: IBGE; 2019.

13. Martins APB, Levi RB, Claro RM, et al. Participação crescente de produtos ultraprocessados na dieta brasileira (1987-2009). Rev. Saúde Pública. 2013 [acesso em $2021 \mathrm{dez} 21$ ]; (47):656-665. Disponível em: https://doi.org/10.1590/S0034-8910.2013047004968.

14. Mendonça RD, Pimenta AM, Gea A, et al. Ultraprocessed food consumption and risk of overweight and obesity: the University of Navarra Follow-Up (SUN) cohort study. Am J Clin Nutr. 2016 [acesso em 2021 dez 21]; 104(5):1433-1440. Disponível em: https://doi. org/10.3945/ajen.116.135004.

15. Instituto Brasileiro de Geografia e Estatística. Pesquisa Nacional por Amostra de Domicílios Contínua (PNAD). Rio de Janeiro: IBGE; 2019.
16. Dias PC, Henriques P, Anjos LA, et al. Obesidade e políticas públicas: concepções e estratégias adotadas pelo governo brasileiro. Cad. Saúde Pública. 2017 [acesso em 2021 dez 21]; 33(7):e00006016. Disponível em: https://doi.org/10.1590/0102-311X00006016.

17. Brasil. Ministério da Saúde, Secretaria de Atenção à Saúde, Departamento de Atenção Básica. Caderno de Atenção Básica 38 - Estratégias para o cuidado da pessoa com doença crônica: obesidade. Brasília, DF: Ministério da Saúde; 2014

18. Brasil. Ministério da Saúde, Coordenação de Gestão de Protocolos Clínicos e Diretrizes Terapêuticas. Protocolo Clínico e Diretrizes Terapêuticas (PCDT) para sobrepeso e obesidade em adultos. Brasília, DF: Ministério da Saúde; 2021. 353 p.

19. São Paulo. Secretaria Municipal de Saúde. Protocolo de Atendimento da Linha de Cuidado de Sobrepeso e Obesidade. São Paulo: SMS; 2020.

20. Bardin L. Análise de Conteúdo. 5. ed. Portugal: Edições 70; 2008.

21. Mendes Gonçalves RBM. Práticas de Saúde: processos de trabalho e necessidades. Cadernos Cefor, 1 . São Paulo: Centro de Formação dos Trabalhadores em Saúde da Secretaria Municipal da Saúde; 1992.

22. Carneiro Junior N, Nascimento VB, Costa IMC. Relação entre Público e Privado na Atenção Primária à Saúde: considerações preliminares. Saúde soc. 2011 [acesso em 2021 dez 21]; 20(4):971-979. Disponível em: https://doi.org/10.1590/S0104-12902011000400014.

23. Burlandy L, Teixeira MRM, Castro LMC, et al. Modelos de assistência ao indivíduo com obesidade na atenção básica em saúde no Estado do Rio de Janeiro, Brasil. Cad. Saúde Pública. 2020 [acesso em 2021 dez 21]; 36(3):e00093419. Disponível em: https://doi. org/10.1590/0102-311X00093419.

24. Laurell AC, Noriega M. Processo de produção e saúde: trabalho e desgaste operário. São Paulo; Hucitec; 1989. 
25. Biff D, Pires DEP, Forte ECN, et al. Cargas de trabalho de enfermeiros: luzes e sombras na Estratégia Saúde da Família. Ciênc. Saúde Colet. 2020 [acesso em 2021 dez 21]; 25(1):147-158. Disponível em: https:// doi.org/10.1590/1413-81232020251.28622019.

26. Lopes MS, Freitas PP, Carvalho MCR, et al. Challenges for obesity management in a unified health system: the view of health professionals. Family Pract. 2021 [acesso em $2021 \mathrm{dez} 21$ ]; 38(1):4-10. Disponível em: https://doi.org/10.1093/fampra/cmaall7.

27. Tarozo M, Pessa RP. Impacto das Consequências Psicossociais do Estigma do Peso no Tratamento da Obesidade: uma Revisão Integrativa da Literatura. Psicol. ciênc. 2020 [acesso em 2021 dez 21]; (40):e190910. Disponível em: https://doi.org/10.1590/19823703003190910.

28. Ferreira L, Barbosa JSA, Esposti CDD, et al. Educação Permanente em Saúde na atenção primária: uma revisão integrativa da literatura. Saúde debate. 2019 [acesso em 2021 dez 21]; 43(120):223-239. Disponível em: https://doi.org/10.1590/0103-1104201912017.

29. Diniz DS, Sá MC. O uso das narrativas e do dispositivo grupal na formação/educação permanente dos profissionais de saúde: uma revisão de literatura. Interface comun. saúde educ. 2019 [acesso em 2021 dez 21]; (23):e180217. Disponível em: https://doi. org/10.1590/Interface.180217.

30. Andrade RS, Caldas LBSN, Falcão MPD, et al. Processo de trabalho em unidade de saúde da família e a educação permanente. Trab. educ. saúde. 2016 [acesso em $2021 \mathrm{dez} 21$ ]; 14(2):505-521. Disponível em: https://doi.org/10.1590/1981-7746-sip00108.

31. Machado PMO, Lacerda JT, Colussi CF, et al. Estrutura e processo de trabalho para as ações de alimentação e nutrição na Atenção Primária à Saúde no Brasil, 2014. Epidemiol. Serv. Saúde. 2021 [acesso em 2021 dez 21]; 30(2):e2020635. Disponível em: http://scielo. iec.gov.br/scielo.php?script=sci_arttext\&pid=S1679$-49742021000200011 \& \operatorname{lng}=p t$.
32. Matuda CCG, Pinto NRS, Martins CL, et al. Colaboração interprofissional na Estratégia Saúde da Família: implicações para a produção do cuidado e a gestão do trabalho. Ciênc. Saúde Colet. 2015 [acesso em 2021 dez 21]; 20(8):2511-2521. Disponível em: https:// doi.org/10.1590/1413-81232015208.11652014.

33. Carnut L, Mendes A. Capital- Estado na crise contemporânea: o gerencialismo na saúde pública. Argum. 2018 [acesso em $2021 \mathrm{dez} 21$ ]; 10(2):108-121. Disponível em: https://doi.org/10.18315/argumentum.v10i2.19528.

34. Carneiro CCG, Martins MIC. Novos modelos de gestão do trabalho no setor público de saúde e o trabalho do agente comunitário de saúde. Trab. educ. saúde. 2015 [acesso em $2021 \mathrm{dez} 21$ ]; 13(1):45-66. Disponível em: https://doi.org/10.1590/1981-7746-sip00029.

35. Teston LM, Mendes A, Carnut L, et al. Avaliação no SUS: uma crítica à ideologia do produtivismo no capitalismo contemporâneo. Saúde debate. 2018 [acesso em 2021 dez 21]; 42(esp3):226-239. Disponível em: https://doi.org/10.1590/0103-11042018S317.

36. Souza HS. A Interpretação do trabalho em enfermagem no capitalismo financeirizado: um estudo na perspectiva teórica do fluxo tensionado. [dissertação]. São Paulo: Faculdade de Saúde Pública, Universidade de São Paulo; 2015.

37. Bourget MMM. Fatores de permanência e desligamento de médicos em serviços de atenção primária à saúde na zona Leste do município de São Paulo. [tese]. São Paulo: Faculdade de Medicina, Universidade de São Paulo; 2019.

Recebido em 30/07/2021

Aprovado em 06/12/2021

Conflito de interesses: inexistente

Suporte financeiro: Coordenação de Aperfeiçoamento de Pessoal

de Nível Superior (Capes). Código de Financiamento 001 